\title{
Neural Network Under External Stimulus: Improving Storage Capacity and Reactions
}

\author{
Evaldo M. F. Curado ${ }^{1,2}$, Nilo B. Melgar ${ }^{1}$, and Fernando D. Nobre ${ }^{1,2}$ \\ ${ }^{1}$ Centro Brasileiro de Pesquisas Físicas and \\ ${ }^{2}$ National Institute of Science and Technology for Complex Systems \\ Rua Xavier Sigaud 150, Rio de Janeiro, Brazil
}

\begin{abstract}
Motivated by the way animals react in nature, we introduce a fullyconnected neural-network model, which incorporates the external pattern presented to the system as a fundamental tool of the recognition process. In this new scenario, in the absence of this external field, memories are not attractors inside basins of attraction, as in usual attractor neural networks, although basins may be created according to the external pattern, thus allowing storing a much larger number of memories. The key point consists in calibrating the influence of the external pattern such as to cancel the noise generated by those memories not correlated with the external pattern. We illustrate how this proposal works by including this new contribution in the standard Hopfield model, showing a significant increase in its recognition capacity (typically by a factor $10^{2}$ ). As an additional feature of this model, we show its ability to react promptly to changes in the external environment, a crucial attribute of living beings. This procedure can be applied to a wide variety of neural-network models to increase considerably their recognition and reaction capabilities.
\end{abstract}

Keywords: Neural networks, Nonlinear Dynamical Systems, Pattern Recognition, Numerical Simulations

Corresponding author: EMF Curado, evaldo@cbpf.br 


\section{Introduction}

The evolution theory, proposed by Darwin and Wallace [1, 2, 3], represents one of the most important theories nowadays, being fundamental to understand our world. It is accepted by all scientists as a starting point to study everything related to living beings, or parts of them. All organs of the living creatures were, of course, molded by evolution. The brain also grew and developed by the action of natural selection. It has to work, actually, respecting its evolutionary history, which means its development, from generation to generation, from species to species, of a complex system with prompt responses to external stimuli, a fundamental condition to survive. This stimuli-dependent working way of the brain should be behind all its tasks, the instinctive ones, as well as those related to cognition.

Neural networks were introduced to model some functions of the brain [4] and their first main practical application was pattern recognition [5]. However, since the proposition by Hopfield, in the beginning of the 1980s [6], the so-called attractor neural networks (ANNs) became paradigmatic and have concentrated the attention of many workers (for good reviews, see textbooks $[7,8,9])$. In the standard Hopfield model, a Hamiltonian can be written, where the strength of the coupling constants are associated with the memories, and based on Hebb's learning rule [10]. The stored memories are the fundamental states of the system, defining a nontrivial phase space, where each memory possesses its own basin of attraction. An initial pattern, presenting an important overlap with one stored memory and then belonging to its basin of attraction, evolves by means of an appropriate dynamics (stochastic or not) to the lower energy state, i.e., to the stored memory, leading to the recognition of that pattern $[11,12,13]$.

Herein we will address two limitations usually found in ANNs: (a) A maximum limit in their storage capacity; (b) Their inefficiency in reacting to changes in the external environment. In what concerns constraint (a), since the memories correspond to ground states, each one presenting its own basin of attraction, the size of the basins dies down as the number of stored memories increases, limiting severely the number of stored memories, even if these memories are not correlated. In general, the storage capacity of an ANN, with $N$ neuronal units, may be expressed as $p=\alpha N$, with $\alpha$ characterized by threshold values $\left(\alpha_{c}\right)$, and in most cases $\alpha_{c}$ is much smaller than unit. In the standard Hopfield model, it is well-known that $\alpha_{c} \simeq 0.14$, so that the system becomes unable to recognize any further pattern for $\alpha>0.14$. 
The limitation in (b) comes from the fact that, once a pattern is recognized, the system stays in that state, even if the input pattern had acted only in the beginning of the process; this feature prevents quick reactions to external changes. Improvements on the Hopfield model, like turning the coupling constants non-symmetric (in which case there is no Hamiltonian) $[14,15,16]$, correlating or coupling memories $[17,18,19,20]$, introducing dilution $[16$, $21,22,23,24,25]$ or autapses [26], attempt to satisfy, at least partially, the drawbacks (a) and (b) above; in what follows, we propose a model to overcome both difficulties.

In Section 2 we introduce the main ideas, the framework, and discuss the effects of an external stimulus for pattern recognition; moreover, we illustrate how the procedure may be implemented for the standard Hopfield model. In Section 3 we define relevant quantities to be investigated and present results from numerical simulations; it is shown that this model yields a significant increase in the storage capacity, and that it is able to react promptly under changes in the external environment. Finally, in Section 4 we present our main conclusions.

\section{Stimulus-dependent model: framework and examples}

The central point of the present proposal concerns a new contribution that takes into account the influence of external stimuli, so important for living beings. Roughly, we express the local field exerted on neuron $i$ as

$$
h_{i}=\text { signal }+ \text { noise }+ \text { external stimulus },
$$

where the first two terms on the r.h.s correspond to the usual signal and noise contributions, produced by other neurons on neuron $i$. The extra term corresponds to the external stimulus, which will be always present during the whole process of recognition. Hence, it is possible to calibrate the influence of the external stimulus, along the time-evolution process, in such a way to cancel, as much as possible, the noise produced by the memories not correlated with the external stimulus. In this framework, the initial state of the neurons becomes irrelevant; the fundamental point now is how to tune appropriately the intensity of the external stimulus so that the signal remains, allowing the recognition of the stimulus, if associated with some 
stored pattern. From now on, we refer to this scheme as a stimulus-dependent neural network (SDNN).

Although this proposal can be applied to large diversity of neural-network models (e.g., an external-stimulus contribution has shown its effectiveness in the suppression of chaos in a fully-connected neural-network model [27]), herein we illustrate how it works on the standard Hopfield model. In this case, the local field $h_{i}$, at time $t$, becomes

$$
h_{i}(t)=\sum_{j \neq i}^{N} J_{i j} \sigma_{j}(t)+\kappa \eta_{i},
$$

with $\sigma_{i}(t)= \pm 1(i=1,2, \cdots, N)$ representing the state of the $i$-th neuron at time $t$ [4], activated or at rest. Moreover, $J_{i j}$ stands for the intensity of the synapses between neurons $i$ and $j$ (considered as symmetric). The second term on the r.h.s. corresponds to the external stimulus on neuron $i$, where $\eta_{i}= \pm 1$ denotes its effect on the $i$-th neuron, which should remain fixed as long as the stimulus is present, and $\kappa(\kappa \geq 0)$ depicts the intensity of the external stimulus considered in the recognition process. The intensity of the synapses $J_{i j}$ can be expressed in terms of the $p$-stored memories $\left\{\xi_{i}^{\mu}\right\}$ as (Hebb's rule)

$$
J_{i j}=\frac{1}{N} \sum_{\mu=1}^{p} \xi_{i}^{\mu} \xi_{j}^{\mu} \quad(i \neq j),
$$

where $\xi_{i}^{\mu}= \pm 1$ for any $i$ and $\mu$. The memories also remain fixed along the whole time evolution and are assumed to be orthogonal on the average [for finite $N$ there may occur overlaps between memories of $O(1 / \sqrt{N})$ ] and following

$$
P\left(\xi_{i}^{\mu}\right)=\frac{1}{2} \delta\left(\xi_{i}^{\mu}-1\right)+\frac{1}{2} \delta\left(\xi_{i}^{\mu}+1\right) .
$$

In what concerns the external stimulus, we will consider two typical situations: (i) It presents an overlap with one specific stored memory (e.g., memory $\rho, 1 \leq \rho \leq p$ ), orthogonal to all other memories; (ii) It is orthogonal to all memories, presenting no overlap with any stored memory. This can be expressed by assuming that the set $\left\{\eta_{i}\right\}$ obeys the probability distribution

$$
P\left(\eta_{i}\right)=\gamma \delta\left(\eta_{i}-\xi_{i}^{\mu}\right)+(1-\gamma) \delta\left(\eta_{i}+\xi_{i}^{\mu}\right)
$$


where $1 / 2<\gamma \leq 1$ for $\mu=\rho$ and $\gamma=1 / 2$ for $\mu \neq \rho$. Clearly, $\gamma=1$ means that the set of external-stimulus signs $\left\{\eta_{i}\right\}$ yields the same pattern as memory $\rho$; this covers frequent real situations, where one should recognize an external pattern that coincides precisely with the stored one. Herein, the main idea is to keep the influence of the external pattern during the whole process, and not only as an initial state, as happens in typical ANNs.

To understand better the role played by the external stimulus, let us analyze further the local field of Eq. (2), separating the contribution of memory $\rho$, from those of other memories $(\mu \neq \rho)$ in Eq. (3). One obtains

$$
h_{i}(t)=\frac{1}{N} \sum_{j \neq i}^{N} \xi_{i}^{\rho} \xi_{j}^{\rho} \sigma_{j}(t)+\frac{1}{N} \sum_{j \neq i}^{N} \sum_{\mu \neq \rho}^{p} \xi_{i}^{\mu} \xi_{j}^{\mu} \sigma_{j}(t)+\kappa \eta_{i},
$$

where one readily identifies each of the contributions defined in Eq. (1). The first one is the signal, when the neuron configuration $\left\{\sigma_{i}\right\}$ is roughly the same as the stored memory $\left\{\xi_{i}^{\rho}\right\}$; the second one represents the noise, induced by other memories distinct from $\rho$; the third contribution corresponds to the external stimulus. In the particular case $\gamma=1$, if we consider $\sigma_{i}(t)=\eta_{i}=\xi_{i}^{\rho}$, then

$$
h_{i}(t)=\frac{N-1}{N} \xi_{i}^{\rho}+\frac{1}{N} \sum_{j \neq i}^{N} \sum_{\mu \neq \rho}^{p} \xi_{i}^{\mu} \xi_{j}^{\mu} \xi_{j}^{\rho}+\kappa \xi_{i}^{\rho},
$$

suggesting that by choosing $\kappa$ appropriately, one may favor the signal contribution, allowing the external stimulus to cancel roughly the noise term, granting the system to recognize the submitted external pattern.

Let us estimate approximately this optimal value of $\kappa$, by analyzing the noise contribution in Eq. (7),

$$
h_{i}^{\text {noise }}(t)=\frac{1}{N} \sum_{j \neq i}^{N} \sum_{\mu \neq \rho}^{p} \xi_{i}^{\mu} \xi_{j}^{\mu} \xi_{j}^{\rho} .
$$

Since the patterns $\mu$ are not correlated with pattern $\rho$ and the sites $i, j$ are independent, each of these contributions may be considered as independent, i.e., \pm 1 with equal probabilities. For large enough values of $N$ and $p$, the average value on the variables $\{\xi\}$ 's, $\left\langle h_{i}^{\text {noise }}(t)\right\rangle_{\xi} \simeq 0$, whereas its associated variance, $\left\langle\left(h_{i}^{\text {noise }}(t)\right)^{2}\right\rangle_{\xi}$, yields

$$
\left\langle\frac{1}{N^{2}} \sum_{j \neq i}^{N} \sum_{\mu \neq \rho}^{p} \sum_{k \neq i}^{N} \sum_{\nu \neq \rho}^{p} \xi_{i}^{\mu} \xi_{j}^{\mu} \xi_{j}^{\rho} \xi_{i}^{\nu} \xi_{k}^{\nu} \xi_{k}^{\rho}\right\rangle_{\xi} \simeq \frac{p}{N}=\alpha
$$


so that the width of the noise distribution, i.e., $\left\langle\left(h_{i}^{\text {noise }}(t)\right)^{2}\right\rangle_{\xi}^{1 / 2} \sim \sqrt{\alpha}$. Hence, for independent patterns, a good choice for $\kappa$ should roughly be $\sqrt{\alpha}$ and our numerical simulations show a good agreement with this choice. This could yield pattern recognition for values of $p$ much higher than those restricted by the upper limit of the Hopfield model. However, in other models where analytical calculations for the optimal value of $\kappa$ are not feasible, it may be possible to estimate this value numerically; in fact, this is an easy task, as will be shown later on.

\section{Numerical simulations: retrieving memo- ries for large values of $\alpha$}

In order to show how the external stimulus can increase substantially the capacity of stored memories, let us first describe details of the numerical procedure and define some important quantities to be used from now on. First, from Eq. (6), since the couplings $\left\{J_{i j}\right\}$ are symmetric, one can introduce a Hamiltonian at time $t$,

$$
\begin{array}{r}
\mathcal{H}(t)=-\sum_{i} h_{i}(t) \sigma_{i}(t)=-\frac{1}{N} \sum_{(i, j)} \xi_{i}^{\rho} \xi_{j}^{\rho} \sigma_{j}(t) \sigma_{i}(t) \\
-\frac{1}{N} \sum_{(i, j)} \sum_{\mu \neq \rho}^{p} \xi_{i}^{\mu} \xi_{j}^{\mu} \sigma_{j}(t) \sigma_{i}(t)-\kappa \sum_{i=1}^{N} \eta_{i} \sigma_{i}(t)
\end{array}
$$

where $\sum_{(i, j)}$ denote sums over all distinct pairs of neurons, corresponding to a fully-connected neural network.

We studied the system defined by Eqs. (2)-(6) through numerical simulations, for $N=10^{4}$ neurons and $p=\alpha N$ stored memories, with several choices for the parameters $\alpha$ and $\gamma$. At first, we set couplings and external stimuli according to Eqs. (3)-(5); being quenched variables, these quantities remained fixed along each time evolution. The initial neuron configuration was taken at random, $\sigma_{i}(0)= \pm 1$ with equal probabilities; these dynamical variables were updated in a sequential way, through a "zero-temperature" dynamics,

$$
\sigma_{i}(t+1)=\operatorname{sign}\left[h_{i}(t)\right] \quad(\forall i)
$$


and each time unit corresponded to this operation on a single neuron. After a sufficiently long time $\left(t^{*} \simeq 10^{2} \mathrm{~N}\right.$, i.e., each neuron being visited $10^{2}$ times), the nearest local minimum of energy was attained, with macroscopic quantities presenting slight fluctuations for $t>t^{*}$.

For the results that follow, we have given special emphasis to the macroscopic superposition (or overlap) of a state $\left\{\sigma_{i}(t)\right\}$ with a given pattern $\left\{\xi_{i}^{\mu}\right\}$ at time $t$,

$$
m_{\mu}(t)=\frac{1}{N} \sum_{i=1}^{N} \xi_{i}^{\mu} \sigma_{i}(t) \quad(\mu=1,2, \cdots, p),
$$

as well as to the the overlap of a state $\left\{\sigma_{i}(t)\right\}$ with the orthogonal externalstimuli signs $\left\{\eta_{i}\right\}$, in the specific case where $\gamma=1 / 2$ for all memories,

$$
m_{\perp}(t)=\frac{1}{N} \sum_{i=1}^{N} \eta_{i} \sigma_{i}(t)
$$

The quantity above represents a macroscopic superposition with an external pattern, not stored and orthogonal to all stored patterns, for which, $\eta_{i}=$ \pm 1 with equal probabilities [cf. Eq. (5)]. Usually, the set $\left\{m_{\mu}(t)\right\}$ may be considered as components of a $p$-dimensional vector,

$$
\vec{m}(t)=\left(m_{1}(t), m_{2}(t), \cdots, m_{\rho}(t), \cdots, m_{p}(t)\right),
$$

and we will focus on $m_{\rho}(t)$, associated with a single condensed pattern and identified in the first term of the Hamiltonian in Eq. (10). All other components $m_{\mu}(t)(\mu \neq \rho)$ appear in the noise contribution of the Hamiltonian, whereas the superposition $m_{\perp}(t)$ appears as its third term.

The results to be presented below were obtained for $t>t^{*}$, i.e., at a local minimum of energy. Then, one can perform the usual averages, so the quantity of Eq. (12), for the particular pattern $\mu=\rho$, leads to

$$
m_{\rho}=\left\langle\frac{1}{N} \sum_{i=1}^{N} \xi_{i}^{\rho}\left\langle\sigma_{i}\right\rangle\right\rangle_{\eta, \xi}
$$

whereas the superposition in Eq. (13) yields

$$
m_{\perp}=\left\langle\frac{1}{N} \sum_{i=1}^{N} \eta_{i}\left\langle\sigma_{i}\right\rangle\right\rangle_{\eta, \xi}
$$



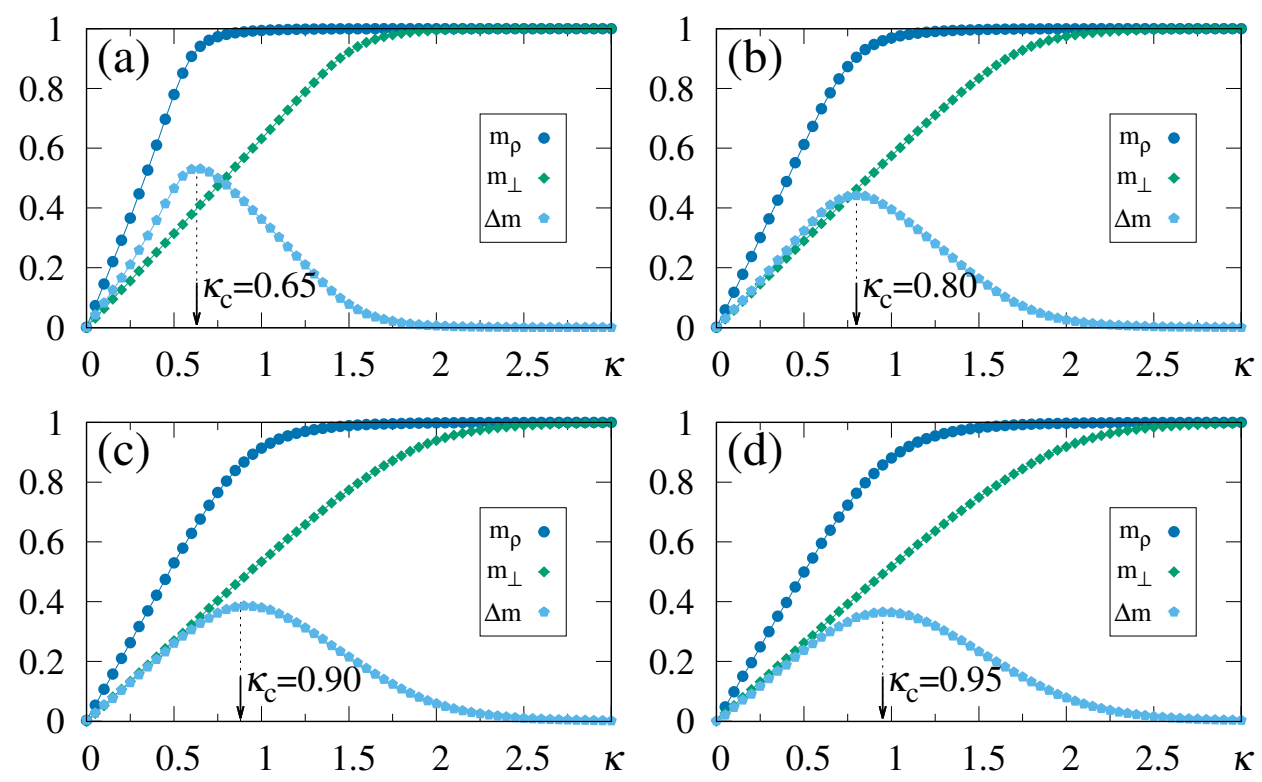

Figure 1: (Color online) Results from numerical simulations, with the quantities of Eqs. (15)-(17) plotted versus $\kappa$, for $\gamma=1.0$, and typical values of $\alpha$ : (a) $\alpha=0.5$; (b) $\alpha=0.7$; (c) $\alpha=0.9$; (d) $\alpha=1.0$. The macroscopic superposition with memory $\rho, m_{\rho}$, is represented by blue circles, the green diamonds are data for $m_{\perp}$, whereas the blue pentagons stand for $\Delta m$. In each case, the maximum value of $\Delta m$ yields the best choice for $\kappa$, denoted by $\kappa_{c}$. The lines interpolating the symbols are guides to the eye.

Above, $\left\langle\sigma_{i}\right\rangle$ correspond to local magnetizations and $\langle. .\rangle_{\eta, \xi}$ stand for averages over different configurations of the quenched variables, for which, following Eqs. (4) and (5), the average on $\left\{\eta_{i}\right\}$ 's should be taken before the one on $\left\{\xi_{i}^{\mu}\right\}$ 's; for these, each simulation was repeated $10^{3}$ times.

Analytical calculations (following the lines of Refs. [11, 12]) were carried to show that the external stimulus may lead to discontinuities in both $m_{\rho}$ and $m_{\perp}$, for $\alpha<0.14$ and given ranges of $\kappa$ (typically $0<\kappa<0.3$ ); however, both parameters exhibited continuous increases with $\kappa$ outside this discontinuity range. Herein we focus on the role played by $\kappa$ in the non-retrieval region, i.e., $\alpha>0.14$, along which one has $m_{\rho}=m_{\perp}=0$ for $\kappa=0$, whereas $\kappa>0$ yields two distinct situations concerning the external pattern, namely, $1 / 2<\gamma \leq 1$ for $m_{\rho}(\kappa)$ and $\gamma=1 / 2$ for $m_{\perp}(\kappa)$; according to this, sufficiently large $\kappa$ lead to $m_{\rho} \rightarrow 2 \gamma-1$ and $m_{\perp} \rightarrow 1$. These two particular limits are not interesting 
for the SDNN; on the other hand, a well-calibrated value of $\kappa$, to be called hereafter $\kappa_{c}$, chosen in such a way to cancel (or diminish as much as possible) the effects of the noise contribution in Eq. (6), represents the crucial point of this new framework. Values $\kappa \ll \kappa_{c}$ will not be sufficient to cancel the noise term, whereas for $\kappa \gg \kappa_{c}$, the external field will dominate the total local field and there will be no pattern recognition at all; in this later case, the local field will simply reproduce the external stimululs, whether associated with a memory or not. Herein, we propose an analysis of the quantity

$$
\Delta m=\left|m_{\rho}-m_{\perp}\right|=\left\langle\frac{1}{N} \sum_{i=1}^{N}\left|\xi_{i}^{\rho}-\eta_{i}\right|\left\langle\sigma_{i}\right\rangle\right\rangle_{\eta, \xi},
$$

in order to identify the optimal value $\kappa=\kappa_{c}$. One notices that only the orthogonal (i.e., non-stored) memories will contribute to $\Delta m$, so that the appropriate choice for $\kappa_{c}$ should correspond to its maximum value, $\max (\Delta m)$.

In Fig. 1 we exhibit results from numerical simulations of the SDNN, where the quantities defined in Eqs. (15)-(17) are plotted versus $\kappa$, for $\gamma=1.0$, and typical values of $\alpha$; notice that all values of $\alpha$ considered are greater (or much greater) than the critical value $\alpha_{c} \simeq 0.14$, above which the standard Hopfield neural network does not recognize any pattern. Moreover, one observes the limits $m_{\rho}=m_{\perp}=0(\kappa=0)$, as well as $m_{\rho} \rightarrow 2 \gamma-1$ and $m_{\perp} \rightarrow 1\left(\kappa \gg \kappa_{c}\right)$, where $\kappa_{c}$ is clearly identified by means of $\max (\Delta m)$. Since the storage capacity $p$ grows with $\alpha$, the variance of the noise contribution also increases, with the analytical approximate result $\kappa_{c} \simeq \sqrt{\alpha}$ [cf. Eq. (9)] showing good agreement with the estimates of Fig. 1. As another consequence of this increase, one finds a tendency towards diminishing the difference $\Delta m$, as well as the magnitude of the values $\max (\Delta m)$.

\subsection{Simulation results: different values of $\gamma$ and $\alpha$}

In Fig. 1 we exhibit results from numerical simulations of the SDNN, where the quantities defined in Eqs. (15)-(17) are plotted versus $\kappa$, for $\gamma=1.0$, and typical values of $\alpha$; notice that all values of $\alpha$ considered are greater (or much greater) than the critical value $\alpha_{c} \simeq 0.14$, above which the standard Hopfield neural network does not recognize any pattern. Moreover, one observes the limits $m_{\rho}=m_{\perp}=0(\kappa=0)$, as well as $m_{\rho} \rightarrow 2 \gamma-1$ and $m_{\perp} \rightarrow 1$ $\left(\kappa \gg \kappa_{c}\right)$, where $\kappa_{c}$ is clearly identified by means of $\max (\Delta m)$. Since the storage capacity $p$ grows with $\alpha$, the variance of the noise contribution also increases, with the analytical approximate result $\kappa_{c} \simeq \sqrt{\alpha}[\mathrm{cf}$. Eq. (9)] 

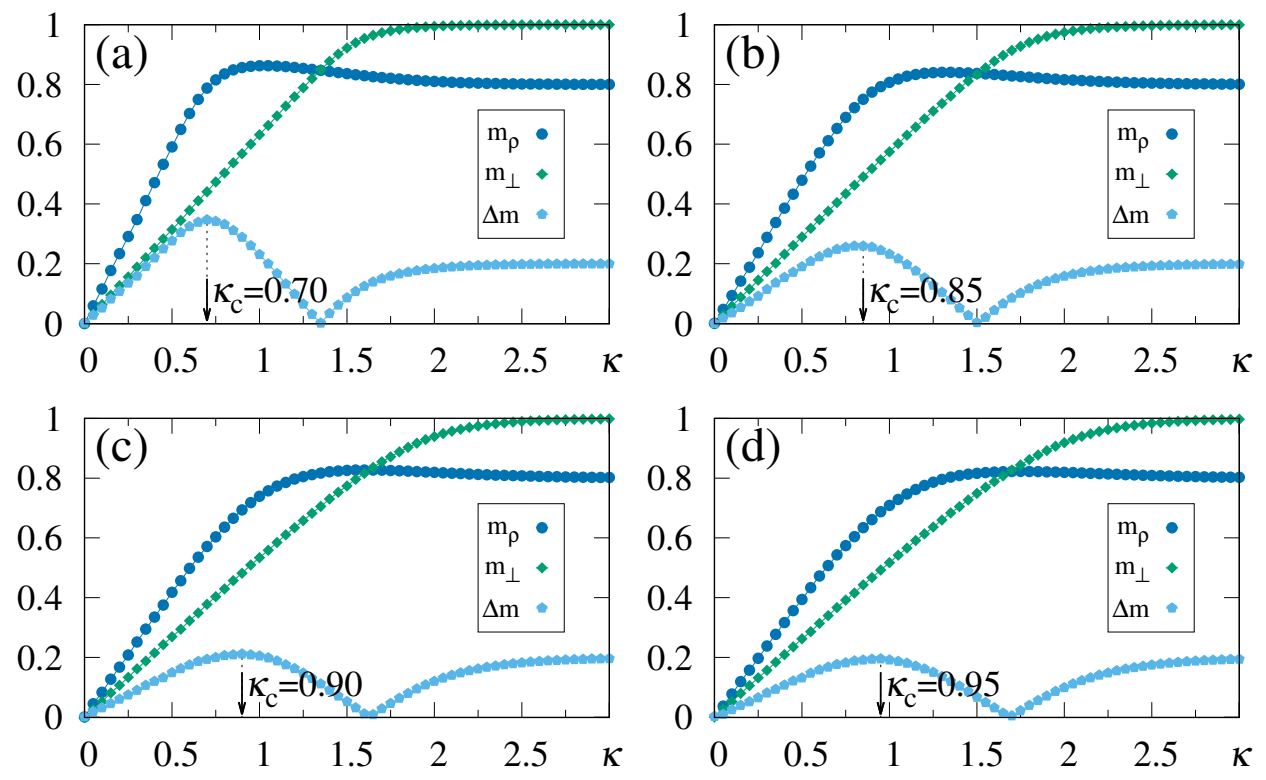

Figure 2: (Color online) Results from numerical simulations, with the quantities of Eqs. (15)-(17) plotted versus $\kappa$, for $\gamma=0.9$, and typical values of $\alpha$ : (a) $\alpha=0.5$; (b) $\alpha=0.7$; (c) $\alpha=0.9$; (d) $\alpha=1.0$. The macroscopic superposition with memory $\rho, m_{\rho}$, is represented by blue circles, the green diamonds are data for $m_{\perp}$, whereas the blue pentagons stand for $\Delta m$. In each case, the maximum value of $\Delta m$ yields the best choice for $\kappa$, denoted by $\kappa_{c}$. The lines interpolating the symbols are guides to the eye.

showing good agreement with the estimates of Fig. 1. As another consequence of this increase, one finds a tendency towards diminishing the difference $\Delta m$, as well as the magnitude of the values $\max (\Delta m)$.

A curious effect is found in Fig. 2, where we present the quantities of Eqs. (15)-(17) versus $\kappa$, for $\gamma=0.9$, and the same values of $\alpha$ used in Fig. 1. For $\kappa<\kappa_{c}$, all plots are qualitatively similar to those of Fig. 1 and particularly, very close estimates for $\kappa_{c}$ (or even equal in some cases). However, for $\kappa \gg \kappa_{c}$ one obtains the limits $m_{\rho} \rightarrow 2 \gamma-1=0.8$ and $m_{\perp} \rightarrow 1$, so that their plots cross $\left(m_{\rho}=m_{\perp}\right)$ at some value of $\kappa$, yielding $\Delta m=0$. To the right of this crossing point one has that $m_{\perp}>m_{\rho}$, signalling that the external stimulus starts dominating the total local field, deteriorating pattern recognition, and for $\kappa \gg \kappa_{c}$ the local field will simply reproduce the external stimululs, whether associated with a memory or not. 

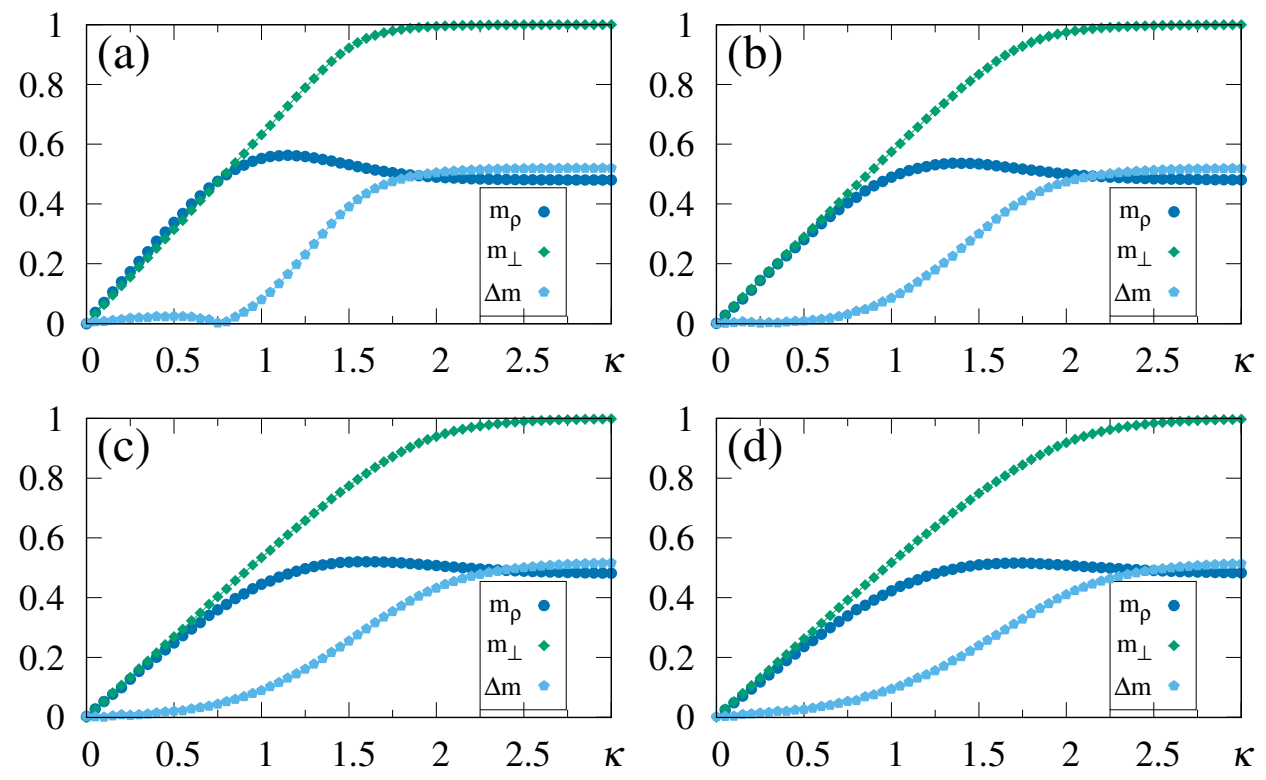

Figure 3: (Color online) Results from numerical simulations, with the quantities of Eqs. (15)-(17) plotted versus $\kappa$, for $\gamma=0.74$, and typical values of $\alpha$ : (a) $\alpha=0.5$; (b) $\alpha=0.7$; (c) $\alpha=0.9$; (d) $\alpha=1.0$. The macroscopic superposition with memory $\rho, m_{\rho}$, is represented by blue circles, the green diamonds are data for $m_{\perp}$, whereas the blue pentagons stand for $\Delta m$. The lines interpolating the symbols are guides to the eye.

The most striking result shown in Figs. 1 and 2 is certainly concerned with the range of $\alpha$ values, above the critical value of the standard Hopfield model, and still allowing pattern recognition easily. In the particular case $\alpha=1$ [cf. Figs. $1(\mathrm{~d})$ and $2(\mathrm{~d})$ ], one finds $\kappa_{c} \simeq 0.95$ in both cases $\gamma=1.0$ and $\gamma=0.9$, with significant values for the macroscopic superposition, $m_{\rho} \simeq 0.9$ [Fig. 1(d)] and $m_{\rho} \simeq 0.7$ [Fig. 2(d)]. As mentioned before, the SDNN presents no basins of attraction, although it creates a huge and single basin of attraction when $\kappa \simeq \kappa_{c}$; however, by setting $\kappa=0$, this basin of attraction disappears and the memories do not occupy any volume in phase space, for any $\alpha>\alpha_{c}$. Within this framework, the recognition works well even for values of $\alpha \gg 1$, as will be shown later.

In Fig. 3 we show the limit of disturbance of the stored pattern for recognition, $\gamma \simeq 0.74$. Essentially, there is not a clear optimal choice for $\kappa$ in this case, so that we can not distinguish a stored pattern from a new pattern, 

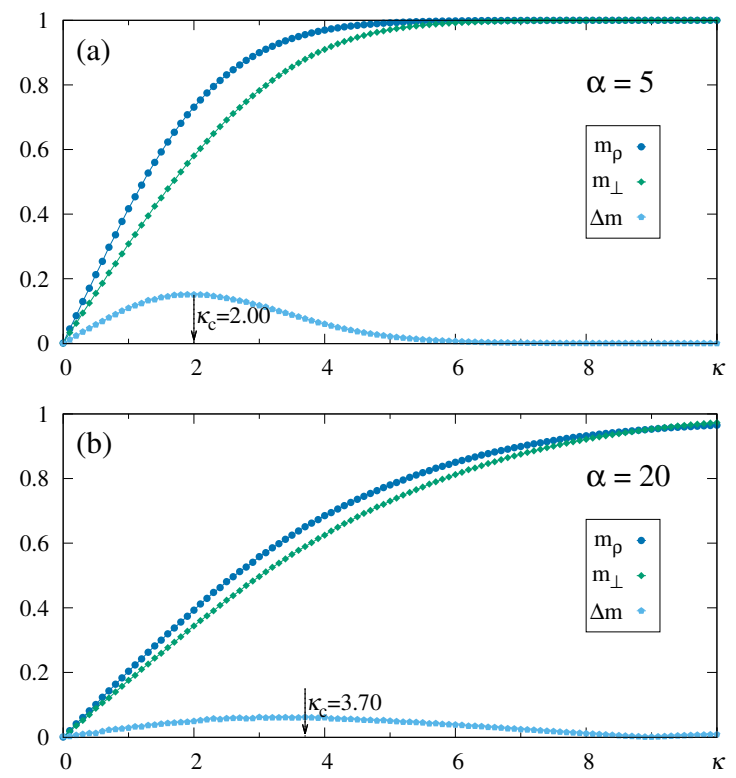

Figure 4: (Color online) Results from numerical simulations, with the quantities of Eqs. (15)-(17) plotted versus $\kappa$, for $\gamma=1.0$, and two choices for $\alpha$ : (a) $\alpha=5$; (b) $\alpha=20$. The macroscopic superposition with memory $\rho, m_{\rho}$, is represented by blue circles, the green diamonds are data for $m_{\perp}$, whereas the blue pentagons stand for $\Delta m$. In each case, the maximum value of $\Delta m$ yields the best choice for $\kappa$, denoted by $\kappa_{c}$. The lines interpolating the symbols are guides to the eye.

not stored and orthogonal to other stored patterns. Hence, in this approach we can deform the stored pattern up to $25 \%$ and the SDNN will still recognize it, even for values of $\alpha$ greater than the critical limit of the Hopfield model, representing an impressive performance, when compared with other neural-network models known in the literature.

Additionally, we have probed much higher values of $\alpha$, in order to test the possibility of a limit for the storage of memories. In Fig. 4 we have chosen two rather large values of $\alpha$, namely, $\alpha=5$ [Fig. 4(a)] and $\alpha=20$ [Fig. 4(b)]; the external pattern remained equal to $\rho$, i.e., $\gamma=1$, like in Fig. 1. In Fig. 4(a) we get $\kappa_{c} \simeq 2.0$, whereas the difference $\Delta m$ remains quite significant (typically, $\Delta m \simeq 0.2$ ), still allowing recognition clearly. However, one should notice the magnitude of $\alpha$ considered in Fig. $4(\mathrm{~b}),\left(\alpha / \alpha_{c}\right) \simeq 143$, when compared with the critical limit of the standard Hopfield neural network. By 


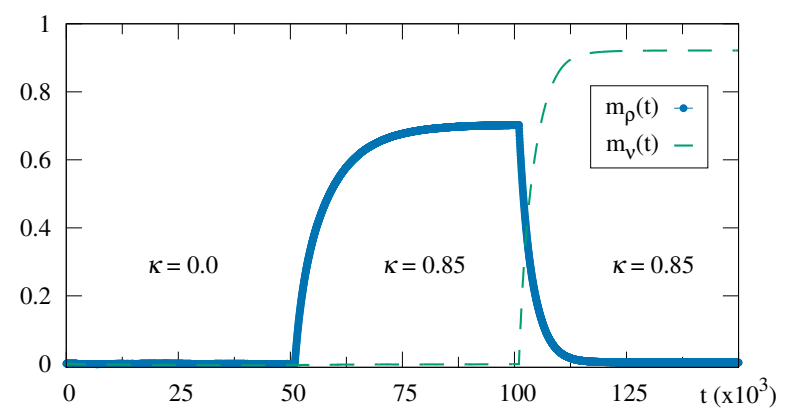

Figure 5: (Color online) The time evolution of the macroscopic superposition $m_{\mu}(t)[$ cf. Eq. (12)] is shown for $\alpha=0.7$, when the external stimulus changes according to Eqs. (18) and (19). The two relevant times are $t_{0}=5.0 \times 10^{4}$ and $t_{1}=10^{5}$, so that for $t<t_{0}$ there is no stimulus, i.e., $\kappa=0.0$. At $t=t_{0}$ a stimulus $(\kappa=0.85)$ appears with a superposition $\gamma_{1}=0.9$ with a stored pattern $\left\{\xi^{\rho}\right\}$, and at $t=t_{1}$ it changes abruptly presenting a superposition $\gamma_{2}=1.0$ with a stored pattern $\left\{\xi^{\nu}\right\}$. The superposition $m_{\nu}(t)$ (dashed green line) remains zero for $t<t_{1}$, whereas $m_{\rho}(t)$ (blue circles) becomes nonzero for $t_{0} \leq t \leq t_{1}$.

analyzing the results of Figs. 1 and 4 one should stress two characteristics, described next. (i) The analytical approximate result (obtained for $\gamma=1$ and $N \rightarrow \infty), \kappa_{c} \simeq \sqrt{\alpha}[\mathrm{cf}$. Eq. (9)], shows a good agreement with most numerical estimates, within $10 \%$ errors, typically, although this discrepancy gets larger (close to 20\%) in the case $\alpha=20$ [Fig. 4(b)]. Such discrepancies are due to the approximations leading to Eq. (9), as well as to the finite sizes used in the simulations. (ii) By increasing $\alpha$ indefinitely, a saturation effect occurs, in the sense that $m_{\perp} \rightarrow m_{\rho}$ from below, yielding $\Delta m \rightarrow 0$. In this regime, clear identifications of $\kappa_{c}$ may become impracticable, explaining the large discrepancy between the analytical and numerical results for $\alpha=20$. In spite of this, we can state that for relatively high values of $\alpha$, as illustrated in Fig. 4(b), the SDNN is able to produce pattern recognition. Nevertheless, we verified that for larger values of $\alpha$ (e.g., $\alpha \gtrsim 40$ ), one gets $\Delta m \rightarrow 0$, so that the identification of an optimal value $\kappa_{c}$ is not possible. 


\subsection{Reactions to changes in the external pattern}

As an additional important attribute of the SDNN, we stress its ability to react under changes in the external environment. Let us then consider that at a time $t_{0}$ we present a pattern associated with one stored memory, say memory $\rho$, and then, at a later time $t_{1}$ we withdraw it, presenting another pattern, associated with memory $\nu$. For living beings, it is crucial to be capable to change the previous recognized pattern, associating the memory $\nu$ to the new pattern, after time $t_{1}$. In order to approach this feature, we allow the set of external-stimulus signs to change in time, i.e., $\left\{\eta_{i}\right\} \equiv\left\{\eta_{i}(t)\right\}$. Furthermore, we assume an abrupt change in these variables at $t=t_{1}$, with the probability distribution in Eq. (5) being replaced by

$$
P\left(\eta_{i}(t)\right)=\gamma_{1} \delta\left(\eta_{i}(t)-\xi_{i}^{\rho}\right)+\left(1-\gamma_{1}\right) \delta\left(\eta_{i}(t)+\xi_{i}^{\rho}\right),
$$

for $t_{0} \leq t \leq t_{1}$, whereas for $t>t_{1}$ one has

$$
P\left(\eta_{i}(t)\right)=\gamma_{2} \delta\left(\eta_{i}(t)-\xi_{i}^{\nu}\right)+\left(1-\gamma_{2}\right) \delta\left(\eta_{i}(t)+\xi_{i}^{\nu}\right),
$$

with $0 \leq \gamma_{1}, \gamma_{2} \leq 1$. In Fig. 5 we illustrate how the SDNN reacts under these changes in the external pattern, by plotting the time-dependent macroscopic superpositions [cf. Eq. (12)] $m_{\rho}(t)$ (blue circles) and $m_{\nu}(t)$ (dashed green line) versus time, for $\alpha=0.7$. Up to $t_{0}=5.0 \times 10^{4}$ no external pattern is presented $(\kappa=0)$, and no memory is recognized. Then, a pattern with $90 \%$ superposition with the stored pattern $\left\{\xi^{\rho}\right\}$ is presented to the system $\left(\gamma_{1}=0.9\right)$ and one notices that the macroscopic superposition $m_{\rho}(t)$ becomes nonzero $\left(0 \leq m_{\rho}(t) \leq 2 \gamma_{1}-1\right)$. Moreover, up to $t_{1}=10^{5}$ the macroscopic superposition $m_{\nu}(t)$ remains zero, becoming nonzero only for $t>t_{1}$, due to a change in the stimulus, where a pattern with a superposition $\gamma_{2}=1.0$ with a stored pattern $\left\{\xi^{\nu}\right\}$ appears, yielding $0 \leq m_{\nu}(t) \leq 1$. At this time, one has an abrupt change in the stimulus and for $t>t_{1}$ one observes another important feature of the SDNN, namely, a decrease in $m_{\rho}(t)$, together with a growth in $m_{\nu}(t)$. One should remind that reactions may also be considered in the standard Hopfield model for $\alpha<\alpha_{c}$, by changing the initial state $\left\{\sigma_{i}(0)\right\}$, which may lead to a jump in phase space to a different basin of attraction. The results of Fig. 5 show clearly that the SDNN modifies the recognized pattern, in a smooth way, according to changes in the external pattern, which is a common behavior in real living beings. 


\section{Conclusions}

We have introduced a fully-connected neural-network model that incorporates the external pattern presented to the system as a fundamental tool of the recognition process. This approach is motivated by the way animals react in nature, always taking into account the external environment. In contrast to usual attractor neural networks, in the absence of the external field, memories are not attractors inside basins of attraction, and basins can be generated for external stimuli presenting significant macroscopic superpositions with stored memories. Such a procedure allows the storage of a much larger number of memories, which can even be several times larger than the total number of neurons $N$. The central point consists in calibrating the influence of the external pattern in order to cancel (as much as possible) the noise generated by memories not correlated with the external pattern.

We have illustrated how this proposal works by including this new contribution in the standard Hopfield model, for which the number of stored memories is given by $p=\alpha N$, and known that for $\alpha \gtrsim 0.14$ it becomes unable to recognize any further pattern. We have verified a significant increase in the recognition capacity of the neural network, so that for this example considered, the range of values of $\alpha$ gets enlarged by a factor $10^{2}$, typically. We have also shown the ability of the model to react promptly to changes in the external environment, which represents a fundamental attribute of living beings that need to react quickly to new external patterns presented, in many situations.

Furthermore, analytical and numerical works under progress have indicated that the above framework improves substantially the recognition capacity in the cases of correlated stored patterns and diluted neural networks. Finally, it is important to mention that this procedure may be implemented to a large diversity of neural-network models, and it will certainly increase considerably their recognition and reaction capabilities, shortening the long bridge separating neural-network models from real living beings.

\section{Acknowledgments}

The authors thank C. Tsallis for fruitful conversations. Partial financial support from CNPq, CAPES, and FAPERJ (Brazilian funding agencies) is acknowledged. 


\section{References}

[1] C. R. Darwin and A. R. Wallace. Journal of the Proceedings of the Linnean Society of London, Zoology, 3 (1858) 45.

[2] C. R. Darwin. On the Origin of Species. John Murray, London, 1859.

[3] G. Beddall Barbara. Journal of the History of Biology, (1968) 261.

[4] W. S. McCulloch and W. Pitts. Bulletin of Mathematical Biophysics, 5 (1943) 115.

[5] W. Pitts W. and W. S. McCulloch. Bulletin of Mathematical Biophysics, 9 (1947) 127.

[6] J. J. Hopfield Proceedings of the national academy of sciences, 79 (1982) 2554.

[7] D. J. Amit. Modelling brain function: the world of attractor neural networks. Cambridge University Press, Cambridge, 1989.

[8] P. Peretto. An introduction to the modelling of neural networks. Cambridge University Press, Cambridge, 1992.

[9] J. A. Hertz, A. Krogh and R.G. Palmer. Introduction to the theory of neural computation CRC Press, Miami, 2018.

[10] D. O. Hebb it The organization of behavior: a neuropsychological theory. Wiley, New York, 1949.

[11] D. J., Amit, H. Gutfreund and H. Sompolinsky. Phys. Rev. A 32 (1985) 1007.

[12] D. J., Amit, H. Gutfreund and H. Sompolinsky. Phys. Rev. Lett. 55 (1985) 1530.

[13] J., McEliece Robert, E. C. Posner, E. R. Rodemich and S. S. Venkatesh. IEEE Transactions on Information Theory, 33 (1987) 461.

[14] B. Derrida and J. P. Nadal. J. Stat. Phys. 48 (1987) 993.

[15] D. A. Stariolo, E. M. F. Curado and F. A. Tamarit. J. Phys. A: Math. Gen. 29 (1996) 4733.

[16] Viola Folli, G. Gost, M. Leonetti and G. Ruocco. Neural Networks, 104 (2018) 50 .

[17] M. V. Tsodyks and M. V. Feigel'man. Europhys. Lett. 6 (1988) 101. 
[18] H. Gutfreund. Phys. Rev. A 37 (1988) 570.

[19] J. Fontanari and W. Theumann. Journal de Physique 51 (1990) 375.

[20] Lucila F. Cugliandolo and M. V. Tsodyks. J. Phys. A: Math. Gen. 27 (1994) 741.

[21] A. Treves and D. J. Amit. J. Phys. A: Math. Gen. 21 (1988) 3155.

[22] M. R. Evans. J. Phys. A: Math. Gen. 22 (1989) 2103.

[23] F. A. Tamarit, D. A. Stariolo and E. M. F. Curado. Phys. Rev. A 43 (1991) 7083.

[24] C. R. da Silva, F. A. Tamarit, N. Lemke, J. J. Arenzon and E. M. F. Curado. J. Phys. A: Math. Gen. 28 (1995) 1593.

[25] C. R. da Silva, F. A. Tamarit and E. M. F. Curado. Phys. Rev. E 55 (1997) 3320 .

[26] G. Gosti, Viola Folli, M. Leonetti and G. Ruocco, Entropy 21 (2019) 726,

[27] K. Rajan, L. F. Abbott and H. Sompolinsky. Phys. Rev. E 82 (2010) 011903. 